\title{
Intake of total dietary sugar and fibre is associated with insulin resistance among Danish 8-10- and 14-16-year-old girls but not boys. European Youth Heart Studies I and II
}

\author{
Iben Kynde ${ }^{1,3, *}$, Nina F Johnsen ${ }^{2}$, Niels Wedderkopp ${ }^{4}$, Ib C Bygbjerg ${ }^{5}$, Jørn W Helge ${ }^{3}$ \\ and Berit L Heitmann ${ }^{1,6}$ \\ ${ }^{1}$ Research Unit for Dietary Studies at the Institute of Preventive Medicine, Copenhagen University Hospital, \\ Centre for Health and Society, Blegdamsvej 3, DK-2200 Copenhagen N, Denmark: ${ }^{2}$ Institute of Cancer \\ Epidemiology, Danish Cancer Society, Copenhagen, Denmark: ${ }^{3}$ Faculty of Health Sciences, Department of \\ Biomedical Sciences, Centre for Healthy Ageing, University of Copenhagen, Copenhagen, Denmark: ${ }^{4}$ Back \\ Research Centre, Institute of Sport Science and Clinical Biomechanics, University of Southern Denmark, Odense, \\ Denmark: ${ }^{5}$ Department of International Health, Immunology \& Microbiology, Copenhagen University, \\ Copenhagen, Denmark: Institute of Sport Science and Clinical Biomechanics, University of Southern Denmark, \\ Odense, Denmark
}

Submitted 3 April 2009: Accepted 20 January 2010: First published online 18 March 2010

\begin{abstract}
Objective: To examine the dietary intake of total sugar, added sugar, non-added sugar and starch as well as dietary fibre and glycaemic index (GI) and their respective associations with insulin resistance.

Design: Mixed linear models were used to study both cross-sectional and prospective associations between carbohydrate components and insulin resistance separately in girls and boys. Diet was assessed by a single $24 \mathrm{~h}$ recall interview and insulin resistance was calculated using the homoestasis model assessment (HOMA). Setting: The Danish part of the European Youth Heart Studies (EYHS) I and II. Subjects: Girls and boys at 8-10 and 14-16 years from EYHS I ( $n$ 651) and 8-10-yearolds from baseline followed up 6 years later in EYHS II ( $n$ 233).

Results: Among girls, a difference in dietary total sugar of $43 \mathrm{~g} / \mathrm{MJ}$ was associated with a $1 \mathrm{SD}$ difference of HOMA and a difference in dietary fibre of $-8 \mathrm{~g} / \mathrm{MJ}$ was associated with a $1 \mathrm{SD}$ difference of HOMA, independent of age, maturity and other confounders (both $P=0 \cdot 03$ ). No baseline associations were found among boys and no prospective associations were found in either sex.

Conclusions: Dietary intake of total sugar may play an adverse role and fibre may play a beneficial role in concurrent insulin resistance among girls but not boys. Sex differences may be due to differences in maturity, physical activity, food patterns and selective reporting behaviours.
\end{abstract}

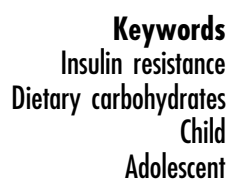

Adolescent
The prevalence of overweight and type 2 diabetes in children has continued to increase since the early $1990 \mathrm{~s}^{(1,2)}$. Controlling obesity and insulin resistance through diet and physical activity is essential in limiting metabolic disturbances and type 2 diabetes ${ }^{(3)}$. The short absorption time that follows the consumption of some dietary carbohydrates may impair blood glucose control, which may result in increased release of non-esterified fatty acids, hyperinsulinaemia ${ }^{(4)}$ and peripheral insulin resistance ${ }^{(5)}$. A diet with a content high in fibre, low in refined sugars with a low-dietary glycaemic index (GI) is required for preventing glucose intolerance, insulin resistance and type 2 diabetes in adults ${ }^{(6)}$. There is a lack of studies on carbohydrate nutrition and insulin resistance among healthy children. Our aim was to study the hypothesis that high intake of total sugar, added sugar and starch, and a high dietary GI as well as low intake of nonadded sugar and dietary fibre in children are associated with insulin resistance concurrently and 6 years later.

\section{Design}

Data are based on the Danish part of the European Youth Heart Studies (EYHS) I and II, a prospective multicentre survey of lifestyle and cardiovascular risk factors in childhood and youth. The studies were carried out from September 1997 to June 1998 and again from September 2003 to June $2004^{(7)}$ and included schoolchildren from twenty-five primary schools in Odense, Denmark. Schools 
were stratified according to the location and socioeconomic status of the geographical area. From each stratum, a two-stage cluster sampling was applied with the schools as the primary unit and the children as the secondary unit. Schools were selected using probability proportional to school size and children were allocated code numbers and randomly selected using random number tables. International standardised procedures were followed and investigators were either health professionals or trained personnel. Parents gave written consent for their child to participate, and children had the option to withdraw from the study at any time. The study followed the principles stipulated in the Helsinki Declaration and was approved by the scientific ethics committee of the local counties of Vejle and Funen, Denmark (VF 20030067).

\section{Participants}

Of 1019 children of predominantly Danish ethnicity, 341 girls and 310 boys from the third (58\%) and ninth (42\%) grades were included in the cross-sectional analyses at baseline. The study population was reduced progressively due to missing data on the following variables as percentage of the eligible population of 1019 children: homoestasis model assessment (HOMA; 9\%), diet (6\%), sexual maturity levels (1\%), body anthropometrics (1\%), cardiorespiratory fitness (6\%), parents' education (13\%) and physical activity (1\%). Follow-up data on serum glucose and insulin existed for 138 girls and 103 boys. The overall participation rates were $75 \%$ at baseline and $65 \%$ at follow-up. The children included in the study were more mature and more active than those excluded at baseline, but not at follow-up, after Bonferroni correction (data not shown).

\section{Insulin resistance}

Blood samples were obtained from an antecubital vein after children had fasted overnight. Samples were frozen at $-80^{\circ} \mathrm{C}$ until further analysis. Serum glucose concentrations were quantified using a hexokinase method and serum insulin concentrations using an enzyme immunoassay method at WHO-certified laboratories at baseline (Bristol, England) and at follow-up (Cambridge, England $)^{(7)}$. Insulin resistance was evaluated using the HOMA: [insulin $(\mu \mathrm{U} / \mathrm{ml}) \times$ glucose $(\mathrm{mmol} / \mathrm{l})] / 22 \cdot 5^{1(8)}$ and standardised to the mean of each sex (Z-scores). That is, the means of HOMA $Z$-scores are defined as zero with a standard deviation of one in each of the study populations of girls and boys.

\section{Dietary intake}

The individual food intake was assessed using one $24 \mathrm{~h}$ recall interview supplemented with a qualitative food record from the same day. Standard measures were visualised with glasses, plates and spoonfuls. The interviews were conducted Monday-Friday and lasted 20-30 min. Third graders were assisted by a parent. Foods were converted into nutrients using the software program Dankost 3000 (Danish Catering Center, Copenhagen, Denmark), referring to national food composition lists ${ }^{(9)}$. The six dietary carbohydrate components were measured as: (i) total sugar comprising all mono- and disaccharides; (ii) added sugar comprising liquid and solid sugars that are not naturally present in foods; (iii) non-added sugar comprising sugars that are naturally present in foods; (iv) dietary fibre comprising soluble and insoluble fibres; (v) starch comprising amylose and amylopectin; and (vi) dietary GI calculated by summing up the GI of each carbohydrate food item $\left(\mathrm{GI}_{i}\right)$ weighted by the specific contribution of each food item ( $i$ ) to the total amount of available carbohydrates (CHO), except dietary fibres: dietary $\mathrm{GI}=\Sigma\left(\mathrm{GI}_{i} \times \mathrm{CHO}_{\mathrm{i}} / \mathrm{CHO}\right)^{(6)}$. GI values were calculated using international tables with white bread equal to $100 \mathrm{GI}$ units $^{(10)}$. Further details are presented elsewhere ${ }^{(11)}$.

\section{Confounding factors}

Children and parents or guardians completed electronic questionnaires with information on sex, date of birth and physical activity level of the child, and educational status of both parents. Physical activity level was categorised as no exercise, occasional exercise or regular exercise. Sexual maturity was assessed using five development stages of breasts or genitals and pubic hair. Children were grouped as prepubertal with Tanner stage 1, pubertal with Tanner stages 2, 3 and 4, and postpubertal with Tanner stage $5^{(12)}$. Height without shoes was measured to the nearest $0.5 \mathrm{~cm}$ and weight with light clothing to the nearest $0 \cdot 1 \mathrm{~kg}$.

\section{Statistical analysis}

Statistical analyses were carried out in the SAS for Windows statistical software package version 9·1 (SAS Institute Inc., Cary, NC, USA). Linear regressions of each carbohydrate component in association with insulin resistance were analysed in cross-sectional and prospective models, stratified by sex. The random effect of primary school was included in the models to counterbalance the effect of schools deriving from the cluster-sampling design. Models were additionally adjusted for the fixed effects of age, BMI, sexual maturity (at both years in prospective analyses), self-reported physical activity and mother's education and other significant carbohydrate components. Statistical significance was determined by a two-sided probability level of $\leq 5 \%$ for the results and $\leq 10 \%$ for selecting confounders.

\section{Results}

Baseline characteristics of anthropometrics, lifestyle and clinical parameters among girls and boys included in cross-sectional and prospective analyses are described in Table 1. Compared with girls, boys were older, more active, had higher serum glucose and consumed more 
Table 1 Baseline characteristics of Danish girls and boys from the European Youth Heart Study I included in cross-sectional analyses and those followed up 6 years later (at 14-16 years) included in the prospective analyses

\begin{tabular}{|c|c|c|c|c|c|c|c|c|}
\hline & \multicolumn{4}{|c|}{ Cross-sectional } & \multicolumn{4}{|c|}{ Prospective } \\
\hline & \multicolumn{2}{|c|}{ Girls (n 341) } & \multicolumn{2}{|c|}{ Boys ( $n$ 310) } & \multicolumn{2}{|c|}{ Girls (n 135) } & \multicolumn{2}{|c|}{ Boys ( $n 98)$} \\
\hline & Mean & SD & Mean & SD & Mean & $\mathrm{SD}$ & Mean & SD \\
\hline Age (years) & $12 \cdot 0^{*}$ & $2 \cdot 9$ & $12 \cdot 5$ & 2.9 & $9 \cdot 6^{*}$ & 0.4 & $9 \cdot 7$ & 0.4 \\
\hline BMl $\left(\mathrm{kg} / \mathrm{m}^{2}\right)$ & $18 \cdot 7$ & $3 \cdot 1$ & $19 \cdot 0$ & 3.0 & $17 \cdot 1$ & $2 \cdot 3$ & $17 \cdot 0$ & 1.9 \\
\hline Physical activity $\dagger$ & $2 \cdot 4^{*}$ & 0.6 & 2.5 & 0.6 & $2 \cdot 4^{*}$ & 0.7 & 2.5 & 0.6 \\
\hline Serum glucose $(\mathrm{mmol} / \mathrm{l})$ & $5 \cdot 1^{*}$ & 0.4 & $5 \cdot 3$ & $0 \cdot 4$ & $5 \cdot 0^{* *}$ & 0.4 & $5 \cdot 2$ & 0.4 \\
\hline Serum insulin $(\mu \mathrm{IU} / \mathrm{ml})$ & $10 \cdot 5$ & $5 \cdot 4$ & $10 \cdot 2$ & $7 \cdot 0$ & $8 \cdot 0$ & $4 \cdot 1$ & $7 \cdot 3$ & $3 \cdot 4$ \\
\hline HOMA (unit) & $2 \cdot 4$ & $1 \cdot 3$ & $2 \cdot 4$ & 1.9 & $1 \cdot 8$ & $1 \cdot 0$ & $1 \cdot 7$ & 0.9 \\
\hline Energy intake (MJ) & $8 \cdot 8^{*}$ & $2 \cdot 2$ & $1 \overline{1} \cdot 1$ & $3 \cdot 6$ & $8 \cdot 6$ & $2 \cdot 0$ & $9 \cdot 0$ & $2 \cdot 1$ \\
\hline Carbohydrate (E\%) & $52 \cdot 6^{*}$ & $7 \cdot 8$ & $51 \cdot 8$ & $7 \cdot 4$ & $51 \cdot 9$ & $7 \cdot 4$ & $12 \cdot 6$ & $6 \cdot 7$ \\
\hline Total sugar (g/MJ) & $12 \cdot 8$ & $4 \cdot 2$ & $12 \cdot 8$ & $4 \cdot 0$ & $12 \cdot 1$ & $4 \cdot 0$ & $12 \cdot 6$ & $3 \cdot 7$ \\
\hline Added sugar (g/MJ) & $6 \cdot 0$ & $4 \cdot 3$ & $6 \cdot 5$ & $4 \cdot 2$ & $5 \cdot 6$ & $4 \cdot 2$ & $6 \cdot 3$ & $4 \cdot 2$ \\
\hline Non-added sugar (g/MJ) & $6 \cdot 7$ & $3 \cdot 1$ & $6 \cdot 3$ & $3 \cdot 0$ & $6 \cdot 5$ & $2 \cdot 7$ & $6 \cdot 3$ & $4 \cdot 2$ \\
\hline Starch $(\mathrm{g} / \mathrm{MJ})$ & $6 \cdot 7$ & $4 \cdot 4$ & $7 \cdot 0$ & $4 \cdot 6$ & $6 \cdot 7$ & $4 \cdot 7$ & $6 \cdot 8$ & $4 \cdot 0$ \\
\hline Fibre (g/MJ) & $2 \cdot 2^{\star \star \star}$ & 0.8 & $2 \cdot 0$ & 0.7 & $2 \cdot 1$ & 0.7 & $2 \cdot 1$ & 0.7 \\
\hline Dietary GI (units) & $84 \cdot 8$ & $6 \cdot 9$ & 83.8 & $7 \cdot 3$ & $85 \cdot 1$ & $7 \cdot 2$ & 83.4 & $7 \cdot 4$ \\
\hline
\end{tabular}

HOMA, homoestasis model assessment; E\%, energy percentage; Gl, glycaemic index.

${ }^{\star} P<0 \cdot 05,{ }^{* \star} P<0.01,{ }^{\star * \star} P<0.0001$ between sex, Student's $t$ test.

†No exercise $=1$, occasional exercise $=2$, regular exercise $=3$.

flndex with white bread $=100$.

total energy, less carbohydrates as a proportion of total energy and less dietary fibre in $\mathrm{g} / \mathrm{MJ}$. However, the only sex difference remaining after Bonferroni corrections was the fibre intake. Among girls, HOMA Z-score was directly associated with total dietary sugar intake and inversely associated with dietary fibre after adjustment for age, sexual maturity, BMI, physical activity, mother's education and school location (Fig. 1). The magnitude of the relationships was that $1 \mathrm{SD}$ difference of HOMA was associated with a difference in dietary total sugar of $43 \mathrm{~g} / \mathrm{MJ}$ and a difference in dietary fibre of $-8 \mathrm{~g} / \mathrm{MJ}$. After Bonferroni correction, results became non-significant. Interactions were found between total dietary sugar intake and $\operatorname{sex}(P=0 \cdot 001)$ as the only carbohydrate component. Among girls, interactions were found between physical activity and dietary intake of total sugar $(P=0 \cdot 001)$ and fibre $(P=0 \cdot 054)$ such that the associations between diet and HOMA $Z$-scores were stronger for girls with a lower rather than a higher activity level. No interactions were identified for grade, sexual maturity or overweight.

Between baseline and follow-up, HOMA increased from $1 \cdot 8(\mathrm{SD} 1 \cdot 0)$ to $2 \cdot 2(\mathrm{SD} 1 \cdot 0)$ units among girls and from $1 \cdot 7$ (sD $0 \cdot 9)$ to $2 \cdot 1$ (sD $1 \cdot 0)$ HOMA units among boys $(P<0 \cdot 05)$. None of the carbohydrate components was associated with HOMA $Z$-scores 6 years later among girls or boys after adjustment for the same confounders as mentioned above and baseline HOMA $Z$-scores and sexual maturity at follow-up (Table 2). In order to study the impact of changes in BMI, physical activity and dietary intake, associations were additionally adjusted for BMI in 2001, physical activity in 2003-2004, and carbohydrate components in 2003-2004 in 117 girls and 82 boys (data not shown). No associations were found, except for a strengthened negative estimate for dietary added sugar among girls compared with the analyses without followup lifestyle data presented in Table 2. This result did not remain significant after Bonferroni correction.

\section{Discussion}

Our study suggests a link between the dietary intake of total sugar and fibre and insulin resistance among girls in a sizeable sample of healthy Danish school children at 8-10 and 14-16 years old. To obtain a decrease in insulin resistance of one standard deviation from either an increased fibre intake or a decreased total sugar intake, a dietary change of 3.5 times the mean intake is required. Dietary changes will normally affect more than one carbohydrate component at one time and findings may still be clinically significant for children with a low fibre intake and a high intake of total sugar. In line with our findings, high intake of total dietary sugar was associated with increased insulin secretion and increased insulin resistance in a high-risk population of overweight Latino adolescents with a family history of type 2 diabetes $^{(13)}$. Previous findings among overweight, adolescent girls found no specific effect of dietary fibre intake on insulin secretion after 12 weeks' lifestyle intervention, independent of added sugar intake ${ }^{(14)}$. Danish girls with an overall fibre-rich diet tended to under-report their total dietary energy intake to a larger extent than girls with a lower dietary fibre intake (data not shown). The results on dietary fibre may therefore be partly explained by dietary-reporting behaviour. No associations were found between dietary GI and insulin resistance. Consistent with our findings, a study using repeated $24 \mathrm{~h}$ interviews found 


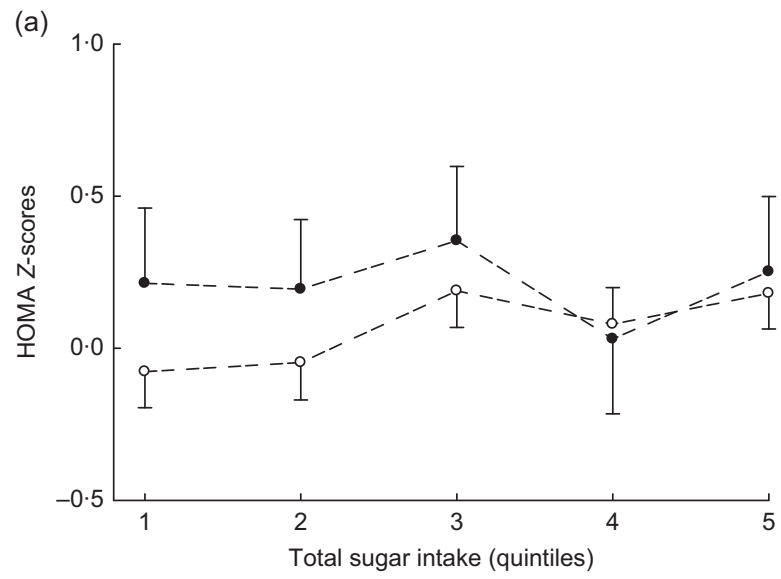

(b)

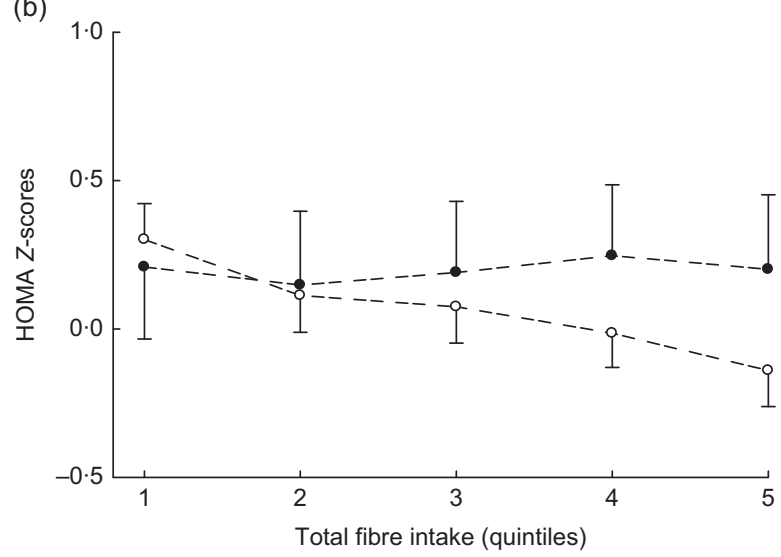

Fig. 1 Insulin resistance measured as HOMA Z-scores of 8-10- and 14-16-year-old girls (-- $--; n$ 341) and boys (-- - - ; $n$ 310) from the Danish part of the European Youth Heart Study I as a function of (a) quintiles of total dietary sugar intake adjusted for primary school, age, sexual maturity, BMI, mother's education, physical activit, and dietary fibre intake and (b) quintiles of total dietary fibre intake adjusted for primary school, age, sexual maturity, BMI, mother's education, physical activity, and total dietary sugar intake. Error bars indicate the SEM

no association between dietary GI and insulin resistance in overweight children with a family history of CVD ${ }^{(13)}$. In the latter study, GI values were assessed using similar international tables to those used in the present study. Another study has found that unadjusted fasting insulin concentrations were directly associated with dietary GI among 8-year-old children ${ }^{(15)}$. However, the study was based on unadjusted analyses and may be confounded by sex, puberty, physical activity or body fatness. We found no association between dietary starch and insulin resistance, and no previous studies have investigated the effect of dietary starch on insulin resistance in children. The cross-sectional results among girls extend prior findings of total dietary sugar to a sample of both normal and overweight children. Sex differences in lifestyle parameters, food compositions and physical activity are likely to explain why we find significant associations among girls and not among boys. Given that girls were less active than boys, girls may have a reduced metabolic flexibility compared with boys, and thus increased susceptibility to the diet.

During the 6 study years, girls progressed from either prepuberty to puberty or puberty to postpuberty, whereas boys progressed from prepuberty to puberty. It has been shown that insulin secretion increases with the onset of puberty, returning to prepubertal levels in postpuberty ${ }^{(16)}$. Although analyses in the present study were adjusted for the maturity stage at baseline and follow-up, it is likely that the non-linear development of insulin resistance has biased the prospective results. Changes in lifestyle from baseline to follow-up did not seem to influence the prospective results. Statistical power was sufficient in the prospective analyses, and the non-significant results may be caused by a smaller variation in the change in insulin resistance between studies than baseline insulin resistance.

Some methodological issues may have influenced the validity of the results of the present study. Both diet and physical activity assessments were based on self-reported data, which may have resulted in selective reporting behaviour. More $24 \mathrm{~h}$ recall interviews for each person could have added to the accuracy of the dietary data, as a single diet recall represents only a snapshot of a child's habitual dietary intake, and therefore, random errors due to day-to-day variations are likely to have blurred true associations. However, another study on diet, obesity and insulin resistance among children showed similarity among the dietary and clinical data of children with a single $24 \mathrm{~h}$ recall interview compared with those with repeated interviews ${ }^{(13)}$. The advantages of the $24 \mathrm{~h}$ recall interview are that the time taken by the child to complete the interview is relatively less and that reading and writing skills are not required. The disadvantages are that the child could have difficulty remembering what and how much they consumed during the last $24 \mathrm{~h}$. The GI assessment may be limited due to a number of reasons. First, the GI assessment was based on food composition tables, comprising primarily Australian and American foods ${ }^{(10)}$. Second, the studies referenced in the tables have been conducted primarily in healthy adults and small sample sizes ${ }^{(17,18)}$ and may not replicate to other groups than healthy adults. Third, the $24 \mathrm{~h}$ interview method was not constructed to distinguish between preparation or processing of foods, e.g. raw, peeled and heated foods, which could have affected the physiological role of starch and other carbohydrate components influencing the $\mathrm{GI}^{(6,19)}$. Although objectively measured physical activity using accelerometers has been assessed in EYHS, we chose to use self-reported activity measures because approximately one-third of the children did not wear the accelerometers and the self-reported measure was more strongly associated with insulin resistance than was the accelerometer measure. Technically, significant 
Table 2 Associations between baseline carbohydrate components and HOMA Z-scores at baseline and at follow-up 6 years later among Danish children from the European Youth Heart Studies I and I. The cross-sectional analyses included 341 girls and 310 boys from the third and ninth grades. The prospective analyses included 135 girls and 98 boys from the third grade who were followed up 6 years later

\begin{tabular}{|c|c|c|c|c|c|c|c|}
\hline & & \multicolumn{3}{|c|}{ HOMA Z-scores (SD) at baseline } & \multicolumn{3}{|c|}{ HOMA Z-scores (SD) at follow-up } \\
\hline & & $\beta^{*}$ & SEE & $P$ value & $\beta^{*}$ & SEE & $P$ value \\
\hline \multirow[t]{2}{*}{ Total dietary sugar $(10 \mathrm{~g} / \mathrm{MJ}) \dagger$} & Girls & 0.23 & $0 \cdot 11$ & 0.03 & -0.07 & 0.23 & $0 \cdot 74$ \\
\hline & Boys & $<-0.01$ & $0 \cdot 13$ & 0.97 & $0 \cdot 18$ & 0.29 & 0.53 \\
\hline \multirow[t]{2}{*}{ Dietary added sugar $(10 \mathrm{~g} / \mathrm{MJ})+$} & Girls & $0 \cdot 20$ & $0 \cdot 11$ & 0.08 & -0.31 & 0.23 & $0 \cdot 18$ \\
\hline & Boys & -0.05 & 0.13 & 0.69 & 0.05 & 0.27 & 0.86 \\
\hline \multirow[t]{2}{*}{ Dietary non-added sugar $(10 \mathrm{~g} / \mathrm{MJ}) \dagger$} & Girls & $0 \cdot 10$ & 0.15 & 0.52 & 0.49 & 0.34 & $0 \cdot 15$ \\
\hline & Boys & 0.08 & $0 \cdot 17$ & 0.63 & 0.22 & $0 \cdot 39$ & 0.57 \\
\hline \multirow[t]{2}{*}{ Dietary fibre $(10 \mathrm{~g} / \mathrm{MJ}) \ddagger$} & Girls & $-1 \cdot 28 \ddagger$ & 0.58 & 0.03 & $-1 \cdot 68$ & $1 \cdot 20$ & $0 \cdot 17$ \\
\hline & Boys & $-0.50 \ddagger$ & 0.71 & 0.48 & $-1 \cdot 18$ & 1.42 & $0 \cdot 41$ \\
\hline \multirow[t]{2}{*}{ Dietary starch $(10 \mathrm{~g} / \mathrm{MJ}) \ddagger, \dagger$} & Girls & $0.02 \ddagger$ & $0 \cdot 10$ & 0.82 & 0.19 & $0 \cdot 19$ & 0.31 \\
\hline & Boys & $0 \cdot 16$ & 0.11 & 0.14 & -0.07 & 0.27 & $0 \cdot 80$ \\
\hline \multirow[t]{2}{*}{ Dietary GI $(10 \mathrm{U}) \ddagger,+, I I$} & Girls & $0 \cdot 01 \ddagger$ & 0.07 & $0 \cdot 82$ & -0.03 & $0 \cdot 12$ & $0 \cdot 80$ \\
\hline & Boys & -0.02 & 0.07 & $0 \cdot 74$ & $-0 \cdot 13$ & $0 \cdot 14$ & $0 \cdot 36$ \\
\hline
\end{tabular}

HOMA, homoestasis model assessment; GI, glycaemic index.

${ }^{*} \beta$ adjusted for age, BMI, sexual maturity (both years at prospective analyses), physical activity and mother's education at baseline and school as school as a random effect.

tAdditionally adjusted for baseline fibre intake.

$\ddagger$ Additionally adjusted for baseline total dietary sugar.

IIIndex with white bread $=100$.

findings could be a result of statistical artefacts due to multiple testing, as $5 \%$ statistical uncertainty is accepted. The diet was not assessed on Fridays and Saturdays, and the actual proportion of dietary added sugar intake may therefore be higher than the measured daily intake in the present study. This might not have affected the findings, as the ranking of individuals is probably the same.

In conclusion, the results suggest that high dietary intake of total sugar and low intake of dietary fibre may be associated with insulin resistance among 8-10-year-old Danish girls but not boys. The cross-sectional finding confirms current recommendations for consuming dietary fibre and minimising added sugar intake. However, the study should be replicated using a more precise diet method. No prospective associations were found between dietary carbohydrate components and insulin resistance. Future research should focus on large-scale, quantitative studies with frequent follow-up intervals during the progression of puberty and more precise diet assessments.

\section{Acknowledgements}

Conflict of interest: The authors have no conflicts of interest. Funding support: N.F.J., B.L.H., I.C.B., N.W. and J.W.H. have nothing to declare. I.K. received grant support from the Danish Heart Foundation, the Aase and Ejnar Danielsen Foundation and Copenhagen University: the Biocampus Research Cluster of Lifestyle-related Illnesses. Authors' contributions: I.K. conducted statistical analyses and wrote the paper. N.F.J., B.L.H., I.C.B., N.W. and J.W.H. helped to conceptualise ideas, interpret findings and review drafts of the manuscript.

\section{References}

1. Molnar D (2004) The prevalence of the metabolic syndrome and type 2 diabetes mellitus in children and adolescents. Int J Obes Relat Metab Disord 28, Suppl. 3, S70-S74.

2. Lobstein T, Baur L \& Uauy R (2004) Obesity in children and young people: a crisis in public health. Obes Rev 5, Suppl. 1, 4-104.

3. Gidding SS, Dennison BA, Birch LL et al. (2005) Dietary recommendations for children and adolescents: a guide for practitioners: consensus statement from the American Heart Association. Circulation 112, 2061-2075.

4. Willett W, Manson J \& Liu S (2002) Glycemic index, glycemic load, and risk of type 2 diabetes. Am J Clin Nutr 76, $274 \mathrm{~S}-280 \mathrm{~S}$

5. Beck-Nielsen H \& Groop LC (1994) Metabolic and genetic characterization of prediabetic states. Sequence of events leading to non-insulin-dependent diabetes mellitus. J Clin Invest 94, 1714-1721.

6. World Health Organization (2003) Diet, Nutrition and the Prevention of Chronic Diseases. Joint WHO/FAO Expert Consultation. WHO Technical Report Series no. 916. Geneva: WHO

7. Riddoch CJ, Edward D, Page AS et al. (2005) The European Youth Heart Study - cardiovascular disease risk factors in children: rationale, aims, study design and validation of methods. J Phys Act Health 2, 115-129.

8. Matthews DR, Hosker JP, Rudenski AS et al. (1985) Homeostasis model assessment: insulin resistance and beta-cell function from fasting plasma glucose and insulin concentrations in man. Diabetologia 28, 412-419.

9. Danish Institute for Food and Veterinary Research, Ministry of Family and Consumer Affairs (2006) Danish Food Composition Databank. Søborg: DFVR.

10. Foster-Powell K, Holt SH \& Brand-Miller JC (2002) International table of glycemic index and glycemic load values: 2002. Am J Clin Nutr 76, 5-56.

11. Nielsen BM, Bjornsbo KS, Tetens I et al. (2005) Dietary glycaemic index and glycaemic load in Danish children in relation to body fatness. Br J Nutr 94, 992-997.

12. Tanner JM (1962) Growth at Adolescence. Oxford: Blackwell. 
13. Davis JN, Alexander KE, Ventura EE et al. (2007) Associations of dietary sugar and glycemic index with adiposity and insulin dynamics in overweight Latino youth. Am J Clin Nutr 86, 1331-1338.

14. Davis JN, Ventura EE, Shaibi GQ et al. (2007) Reduction in added sugar intake and improvement in insulin secretion in overweight latina adolescents. Metab Syndr Relat Disord $\mathbf{5}$, 183-193.

15. Scaglioni S, Stival G \& Giovannini M (2004) Dietary glycemic load, overall glycemic index, and serum insulin concentrations in healthy schoolchildren. Am J Clin Nutr 79, 339-340.
16. Moran A, Jacobs DR Jr, Steinberger J et al. (1999) Insulin resistance during puberty: results from clamp studies in 357 children. Diabetes 48, 2039-2044.

17. Wolever TM \& Bolognesi C (1996) Prediction of glucose and insulin responses of normal subjects after consuming mixed meals varying in energy, protein, fat, carbohydrate and glycemic index. J Nutr 126, 2807-2812.

18. Chew I, Brand JC, Thorburn AW et al. (1988) Application of glycemic index to mixed meals. Am J Clin Nutr $\mathbf{4 7}$, 53-56.

19. Bjorck I, Granfeldt Y, Liljeberg $\mathrm{H}$ et al. (1994) Food properties affecting the digestion and absorption of carbohydrates. Am J Clin Nutr 59, 699S-705S. 